Mots. Les langages du politique

102 | 2013

Les discours sur l'enseignement supérieur et la recherche

\title{
Le discours promotionnel des universités européennes. Homogénéité dans la compétitivité?
}

The promotional discourse of European universities. Homogeneity within competitiveness?

El discurso de promoción de las universidades europeas. ¿Homogeneidad dentro de la competitividad?

Jeoffrey Gaspard

\section{OpenEdition}

Journals

Édition électronique

URL : http://journals.openedition.org/mots/21281

DOI : $10.4000 /$ mots. 21281

ISSN : 1960-6001

Éditeur

ENS Éditions

Édition imprimée

Date de publication : 2 septembre 2013

ISBN : 978-2-84788-414-2

ISSN : 0243-6450

Référence électronique

Jeoffrey Gaspard, « Le discours promotionnel des universités européennes. Homogénéité dans la compétitivité ? », Mots. Les langages du politique [En ligne], 102 | 2013, mis en ligne le 02 septembre 2015, consulté le 01 mai 2019. URL : http://journals.openedition.org/mots/21281 ; DOI : 10.4000/ mots.21281 


\section{Le discours promotionnel des universités européennes. Homogénéité dans la compétitivité?}

Les campus universitaires européens sont actuellement le théâtre de profondes mutations qui concernent tant l'administration journalière des institutions que leurs activités traditionnelles d'enseignement, de recherche et de services à la communauté. Avec pour toile de fond le processus de Bologne et la stratégie de Lisbonne ${ }^{2}$, les discours constituants, massivement produits par les acteurs socio-politiques européens (Commission, OCDE, EUA3, etc.) vantent les mérites d'une refondation des universités : en raison d'évolutions socio-économiques importantes, notamment la mondialisation 4 , celles-ci, pour maintenir un avantage compétitif, sont désormais censées se repositionner en tant qu'actrices incontournables d'une économie de la connaissance globalisée jugée inévitable et sont priées de s'ouvrir à de nouveaux partenaires, collaborer plus activement avec les entreprises, répondre aux demandes de flexibilité des formations d'un monde professionnel en perpétuelle mutation, viser des publics de plus en plus diversifiés et se rendre compétitives sur les plans régional, national et international pour attirer les meilleurs contrats de recherche, les meilleurs scientifiques et étudiants (voir, par exemple, Bruno, 2008, Bruno, Clément et Laval, 2010 et Cussó, 2008).

Cette redéfinition du rôle des universités se fonde sur un impératif de concurrence, principe «universel» étendu à tous les domaines (Dardot, Laval, 2010, p.152) ayant acquis le statut de «norme suprême » des politiques européennes

1. Le processus de Bologne a pour objectif d'harmoniser l'architecture du système européen d'enseignement supérieur en promouvant : 1) la mise en place de trois cycles d'études BA / MA / DOC, dont le but est d'accroître la comparabilité des formations, 2) l'instauration du principe des crédits ECTS qui permet d'établir des équivalences de cursus et enfin 3) la création d'un supplément au diplôme qui décrit en détail le parcours de son détenteur.

2. La stratégie de Lisbonne, dans laquelle est intégré le processus de Bologne, vise à faire de l'Union européenne "l'économie de la connaissance la plus compétitive et la plus dynamique du monde d'ici à 2020» (Conclusions de la présidence du Conseil européen de Lisbonne des 23 et 24 mars 2000).

3. European University Association.

4. Les italiques signalent tout au long de cet article des mots et expressions provenant des (hyper) textes analysés (sites web universitaires ou textes officiels européens - voir ci-dessous).

Université Libre de Bruxelles, Centre de recherche ReSIC jgaspard@ulb.ac.be 
(Bruno, Clément, Laval, 2010, p.11), y compris celles relatives à l'enseignement supérieur et à la recherche. Corrélativement, ce nouveau paradigme implique l'émergence de pratiques inédites au sein des universités : amplification des évaluations de l'enseignement et de la recherche (notamment via le benchmarking 5 et autres mesures quantitatives favorisant la comparaison de performances entre institutions), internationalisation des activités (notamment au niveau du recrutement et des échanges d'étudiants et chercheurs), adoption de pratiques managériales au sein des administrations (par exemple, implémentation d'audits visant l'amélioration de la gouvernance dans le but de réorganiser l'Université pour la rendre dynamique et capable de répondre aux exigences de l'économie de la connaissance), investissement dans les technologies de l'information et de la communication (notamment l'université virtuelle qui permet, entre autres, de cibler un public étudiant varié et disloqué, mais surtout en demande de flexibilité), attention accrue à la qualité ${ }^{6}$ des enseignements (les universités devant s'adapter à la réalité de nouvelles normes en matière de qualité de leurs services, autant que les entreprises en matière de qualité des produits), etc. Ce faisant, de nouvelles dénominations7 circulent et cristallisent cette nouvelle conception de l'Université.

\section{Communication des universités}

Dans un environnement devenu fortement concurrentiel, les activités de communication, calquées sur celles pratiquées en entreprise, occupent une place de plus en plus importante au sein des universités. Avec Defays (2009, p.13), nous suggérons que ce nouveau contexte

[...] a développé [...] l'usage du discours de communication, non seulement entre les différentes catégories du personnel universitaire, mais aussi avec le monde extérieur où l'université doit valoriser son image et multiplier ses contacts [...]. L'université-tour d'ivoire, qui pratiquait des discours réservés aux initiés, a laissé la place à l'université médiatique, qui communique, qui rayonne, qui séduit.

Si la communication institutionnelle se décline en de nombreux artefacts, elle se pratique aujourd'hui prioritairement en ligne. Le site web, en tant que

5. Originellement, le benchmarking est une technique managériale consistant à comparer, à l'aide de chiffres et indicateurs, les pratiques d'entreprises rivales jugées plus « performantes » afin de s'en inspirer.

6. L'Agence pour l'évaluation de la qualité de l'enseignement supérieur (AEQES) en Belgique francophone stipule sur son site web que «l'objectif [des évaluations] est d'améliorer progressivement les pratiques d'enseignement en mettant en évidence les bonnes pratiques, les insuffisances et les problèmes à résoudre et en suscitant des propositions à adresser aux responsables politiques en vue d'améliorer la qualité globale de l'enseignement supérieur en Communauté française».

7. Par exemple, certains chercheurs en management parlent de corporate university, service university ou encore d'entrepreneurial university. 
"vitrine institutionnelle» (Barats, 2009), constitue ainsi, depuis la fin des années quatre-vingt-dix, un dispositif relationnel et promotionnel indispensable géré par les services de communication universitaires. Destiné à de multiples publics, fragmentés et internationaux, le site web institutionnel « reflète le positionnement voulu et l'identité de l'entreprise » (Libaert, Johannes, 2010, p. 108). À ce titre, l'étude du discours qui s'y trouve cristallisé nous semble intéressante, étant donné que les hypertextes promotionnels publiés constituent des artefacts s'inscrivant nécessairement dans un contexte sociohistorique qui leur est propre (c'est-à-dire l'européanisation et le processus de mise en concurrence de l'enseignement supérieur en Europe). Nous nous attendons donc à ce qu'ils portent les traces de ce contexte et suggérons que l'étude de ces traces, et du degré de leur appropriation, permet de porter un éclairage original sur la façon dont les institutions universitaires envisagent et promeuvent le type d'enseignement supérieur qu'elles se proposent de servir.

De manière exploratoire, nous proposons, dans un premier temps, de caractériser les hypertextes promotionnels universitaires en tant que genre de discours à part entière, avant de présenter plus précisément trois types de constantes qui transparaissent systématiquement (c'est-à-dire l'usage récurrent de la formule excellence, la répétition d'unités lexicales spécifiques et la régularité des destinataires ciblés) et dont nous suggérons qu'elles sont à relier au contexte sociodiscursif dans lequel sont ancrées les universités sondées.

\section{Généricité des hypertextes promotionnels}

Le site web universitaire ${ }^{8}$, bien qu'il ne vise pas uniquement la promotion de l'institution (certains modules étant par exemple dédiés à la consultation des catalogues de cours, aux inscriptions en ligne ou à la diffusion des résultats d'examens, qui sont autant de fonctionnalités caractéristiques de l'Université virtuelle), comprend des hypertextes que nous proposons de ranger parmi les genres de discours promotionnels au même titre que les catalogues, brochures ou prospectus.

Construire et valoriser en ligne l'identité institutionnelle d'une université se révèle être une pratique techno-discursive 9 fortement réglée : les constantes

8. Dans le cadre de cette recherche, nous proposons de circonscrire le "site web universitaire» selon un critère purement technique : est considéré comme tel l'ensemble des pages web appartenant à un même nom de domaine (par exemple, les pages reprises sous unistra.fr pour le domaine de l'université de Strasbourg).

9. Le terme signifie ici que communiquer en ligne est une pratique à la fois discursive (« outil» langagier) mais également technique («outil» informatique). Les contraintes constitutives de cette interaction discours/technique font émerger des configurations (hyper)textuelles précises qui se trouvent être en partie caractéristiques du genre de discours analysé. 
repérées à travers le corpus ${ }^{10}$ en témoignent. Au niveau structurel, par exemple, les sites web affichent une relative similitude, notamment en pages d'accueil : celles-ci comprennent généralement un bandeau transversal, affichant la dénomination de l'université ainsi que son logotype, situé en haut de page, et consacrent systématiquement des espaces dédiés aux actualités (qui peuvent concerner à la fois la recherche ou la vie universitaire) et activités récentes (agenda). Les pages d'accueil, à l'aide des segments hypertextuels les énumérant, canalisent également chacun des destinataires précis qu'elles entendent cible ${ }^{11}$ (voir ci-dessous) vers les différents modules contenant de l'information qui leur est destinée. Des régularités se repèrent également au niveau des modules - ou rubriques - disponibles : il s'agit notamment des modules de présentation de l'institution, des formations, de la recherche, du caractère international de l'institution et de la vie étudiante, mais également des pages consacrées aux relations avec les entreprises et à l'insertion professionnelle des diplômés, à la documentation/bibliothèques, aux actualités, aux ressources humaines/recrutement ou encore des pages destinées aux relations médias/presse. En outre, on retrouve, au sein de chaque module, différents niveaux d'organisation récurrents : par exemple, dans le cas des modules de présentation de l'institution, on observe invariablement des pages consacrées aux chiffres et indicateurs relatifs à l'établissement, à sa structure organisationnelle, à la présentation de sa politique générale et de ses missions, à son histoire et à son implantation territoriale.

À ces constantes structurelles et thématiques s'ajoutent également des constantes d'ordre discursif. Ainsi, d'un point de vue énonciatif, les énoncés promotionnels sont généralement pris en charge par un énonciateur omniscient, sans aucune marque de subjectivité et, contrairement au discours promotionnel des entreprises, ne comprennent aucune occurrence du pronom personnel nous qui référerait aux responsables universitaires. Quelquefois, cependant, l'énonciateur interpelle directement le ou les coénonciateur(s) :

(1) Choisir l'Université de Limoges c'est faire le choix d'une université à taille humaine à la pointe de l'innovation. [...] Vous pourrez bénéficier d'un accompagnement dès votre entrée à l'université jusqu'à la fin de vos études. (Hypertexte de l'université de Limoges)

10. Le corpus exploratoire sur lequel nous nous sommes basé est constitué de 18 sites web institutionnels (plus particulièrement les textes extraits des modules hypertextuels présentant l'institution, généralement intitulés «À propos de l'université », «Université », «Institution», etc.) des universités suivantes : Blaise Pascal Clermont-Ferrand, Nice Sophia Antipolis, Strasbourg, Bretagne Occidentale, Pau et Pays de l’Adour, Paris-Est Créteil Val de Marne, Rennes 1, Rennes 2, Limoges, Cergy-Pontoise, Paris 8, Perpignan, Paris 1 Panthéon-Sorbonne, Poitiers, Méditerranée Aix-Marseille 2, François Rabelais Tours, Liège et Lausanne.

11. On retrouve généralement des formulations du type «Je suis... : étudiant / chercheur / journaliste... », accompagnées d'hyperliens vers les pages correspondantes. 
D’un point de vue syntaxique, les hypertextes sont généralement composés d'énoncés courts, construits à partir de phrases assertives mobilisant surtout le présent de l'indicatif et caractérisées par l'usage abondant d'appositions ${ }^{12}$. Les phrases négatives et les points d'exclamation sont quasi absents du corpus. D'un point de vue lexical, on retrouve des noms (atout, ambition, vocation...), adjectifs évaluatifs (incontournable, privilégié, reconnue...) et verbes (encourager, favoriser, promouvoir...) répétés dans le corpus. Enfin, des constructions et expressions récurrentes sont propres au genre du catalogue (emploi d'énumérations, de listings et de termes tels que offre, éventail, gamme, etc.) et d'autres relèvent du discours managérial (emploi de termes et syntagmes tels que gestion, prioritaire, stratégique, génère des revenus, crée des emplois, outils professionnels, structurer une offre, réseaux, évaluation, qualité, benchmarking, spin-off ${ }^{13}$, production).

Ces exemples de régularités nous poussent donc à considérer les hypertextes promotionnels en tant que genres de discours à part entière, si l'on considère, avec Krieg-Planque et Oger (2010), que les genres «matérialisent des formes routinisées ${ }^{14}$ de l'écriture en contexte contraint et portent la trace des enjeux sociopolitiques et institutionnels qu'ils engagent».

Un autre type de régularités caractérise enfin ces hypertextes : il s'agit des relations interdiscursives tissées avec les discours européens sur l'enseignement supérieur. Nous proposons de traiter plus amplement cette dernière observation.

\section{Hypertextes promotionnels et interdiscours européen}

Avec Maingueneau (1984, p.5), nous considérons que l'étude d'un discours spécifique, entendu comme «une dispersion de textes que leur mode d'inscription historique permet de définir comme un espace de régularités énonciatives», passe nécessairement par l'appréhension des autres discours qui entrent en résonance avec le premier. Le concept d'interdiscours permet de rendre compte de ces enchevêtrements. Ainsi, nous suggérons plus

12. Ainsi, la structure prototypique de ces énoncés pourrait être : «[apposition], l'universitéX/elle [verbe à la troisième personne du singulier de l'indicatif présent] [complément du verbe ou attribut du sujet]».

13. Une spin-off, dans le contexte universitaire, est une structure entrepreneuriale, généralement créée au sein de laboratoires, dont l'objectif est de valoriser les résultats des recherches par des brevets, la commercialisation de produits, etc.

14. Les auteurs considèrent que certains « discours institutionnels» (par exemple le compte rendu analytique des débats à l'ONU ou le signalement d'enfant en danger) sont soumis à une « raréfaction des énoncés possibles [qui] recouvre également des phénomènes génériques et textuels liés à l'organisation du discours, aux modalités de l'argumentation ou à des formats rédactionnels ». La production de certains genres de discours est tellement contrainte qu'elle en devient routinière pour leurs producteurs. 
fondamentalement que le processus précis de construction discursive de la promotion institutionnelle des universités passe par l'activation d'éléments discursifs exogènes, c'est-à-dire inscrits dans des discours qui n'ont pas été originellement produits au sein des institutions universitaires sondées. Nous considérerons ci-après trois types de ces relations interdiscursives qui mettent en résonance le discours promotionnel (en ligne) des universités avec les discours constituants européens sur l'enseignement supérieur, et en particulier les nombreuses "communications» au Conseil et/ou aux autres institutions européennes publiées par la Commission ${ }^{15}$.

\section{Formule de l'excellence}

Excellence constitue peut-être une des lexies les plus emblématiques des textes européens consacrés à la refondation des universités. Par exemple, dans un de ces textes ${ }^{16}$ où l'on parle pour la première fois de l'excellence en tant que principe fondamental de la politique communautaire en matière d'enseignement supérieur et de recherche, la Commission (2003) stipulait :

L'Europe a besoin d'excellence dans ses universités, pour optimiser les processus qui sous-tendent la société de la connaissance et pour atteindre l'objectif fixé par le Conseil européen à Lisbonne [...]. Le Conseil européen a admis ce besoin d'excellence en invitant à faire des systèmes européens d'enseignement et de formation une « référence de qualité mondiale » d’ici à 2010.

Cet impératif d'excellence, qui serait garant d'une meilleure compétitivité pour les institutions aux niveaux régional, national mais également international, se retrouve abondamment recontextualisé dans le corpus. Voici, par exemple, trois occurrences de ce terme, repris avec leur cotexte:

(2) L'Université du Luxembourg (UL), institution de petite taille à rayonnement international, vise l'excellence en recherche et en formation.

(3) L'ensemble des formations préparées à l’Université de Poitiers s'appuie sur une recherche d'excellence.

(4) Riche de huit siècles d'excellence, l'Université Paris 1 Panthéon-Sorbonne est

l'une des plus grandes universités françaises.

Ànotre avis, excellence peut, dans ce cas, prétendre au statut de «formule» telle que définie par Krieg-Planque (2009). Pour rappel, une formule 1) présente un caractère figé, ce qui favorise sa mise en circulation, 2) s'inscrit dans une dimension discursive (elle n'existe pas sans les usages que l'on en fait), 3) fonctionne comme un référent social évoquant quelque chose pour tous à

15. Pour une analyse de ces communications de la Commission européenne, voir, entre autres, Cussó (2008).

16. La communication s'intitule Le rôle des universités dans l'Europe de la Connaissance. 
un moment donné de la vie sociale et 4) comporte un aspect polémique qui cristallise des enjeux sociopolitiques.

Un signe de son caractère formulaire réside dans l'emploi du nom excellence au détriment de l'adjectif excellent, qui ne présente aucune occurrence dans le corpus. Le caractère figé de la formule (1) se retrouve donc attesté : on ne parle pas d'universités excellentes mais bien d'universités d'excellence, la deuxième formulation renvoyant alors précisément à la politique générale prônée par la Commission. En ce qui concerne le discours promotionnel, la valeur discursive de la formule (2) se précise : il s'agit d'inscrire la valorisation des universités promues dans le cadre de l'excellence, c'est-à-dire d'insister sur la qualité des services proposés. Or, rien n'est moins précis que ce vers quoi l'excellence renvoie en tant que "référent social» (3), ce qui favorise paradoxalement la mise en circulation du terme et sa mobilisation dans les arguments de promotion. Dans les années quatre-vingt-dix, Readings (1996, p. 22) envisageait déjà le «discours de l'excellence» naissant au sein des universités américaines et remarquait qu'en tant que "principe fédérateur, l'excellence a l'avantage particulier [...] de ne référer à rien (non-referential) » (notre traduction). Plus loin, il ajoutait : "C'est ce manque de référence qui permet à l'excellence de fonctionner comme un agent de traduction (translatability) entre des idiomes radicalement différents : des parkings et des bourses de recherche peuvent être tous deux excellents, et leur excellence ne dépend pas de qualités ou effets spécifiques qu'ils partageraient» (notre traduction). En somme, l'excellence serait une abstraction telle qu'elle pourrait venir se greffer sur n'importe quel support. Ce faisant, l'excellence semble alors un état d'esprit réputé caractéristique de l'institution, plutôt qu'une qualité concrète (et mesurable) attribuée aux objets qu'elle caractérise (d'où l'absence de l'adjectif). Enfin, le caractère polémique (4) de cette formule est également prégnant : si le terme se doit d'être mobilisé dans le discours promotionnel, il n'est pas nécessairement réapproprié par tous les acteurs de la même manière et avec le même engouement. Les différents mouvements de contestation déclenchés sur les campus européens (tels que Slow Science ou les nombreux appels à la désexcellence) ${ }^{17}$ témoignent en effet des enjeux polémiques que cette formule cristallise.

\section{Termes répétés}

En plus d'excellence, les textes européens mobilisent toute une série de termes spécifiques au schème discursif sous-tendant la promotion d'une refondation des universités (Cussó, 2008) : ouverture, service, partenaire, mobilité, compétence, international, qualité, réseau, entreprise, etc. Or, ces termes

17. Le néologisme «désexcellence», à l'instar de «décroissance», est un concept contestataire visant le rejet de la compétitivité fondée justement sur le principe de l'excellence. 
circulent ${ }^{18}$ également dans les hypertextes promotionnels étudiés et constituent autant d'échos intertextuels entre les deux discours, comme le suggèrent les exemples suivants.

Ainsi, l'adéquation entre les compétences et les besoins du monde socioprofessionnel se trouve être valorisée quand il s'agit de décrire l'attrait de compétences acquises dans le cadre d'une formation spécifique à l'université de Lausanne :

(5) Pour ceux qui souhaitent acquérir une formation complémentaire, les postgrades (Masters of advanced studies et écoles doctorales) permettent de s'intégrer de façon ciblée dans le marché du travail ou de développer des compétences dans un domaine connexe.

L'université Blaise Pascal Clermont-Ferrand proclame, quant à elle :

(6) Les enquêtes d'insertion professionnelle, accompagnées de la déclinaison de nos diplômes en termes de compétences, seront une source d'information précieuse pour contribuer à une meilleure orientation et insertion professionnelle.

La mobilité constitue également un thème largement utilisé pour valoriser les institutions : on fera principalement référence à la mobilité des étudiants et des chercheurs, qui est / doit être accrue, augmentée, encouragée, favorisée, renforcée, soutenue, promue, etc. L'université de Perpignan stipule, par exemple, qu'elle

(7) [...] soutient une politique volontaire de mobilité et d'accueil conduisant : à internationaliser ses formations, à amplifier encore les activités de recherche qui se développent avec une participation forte aux programmes européens, à des projets intégrés et des réseaux d'excellence.

L'université de Liège fait même de la mobilité une valeur:

(8) Sa vocation pluraliste, son ouverture sur les réalités politiques, sociales, technologiques et industrielles, sa participation active aux programmes internationaux et européens de recherche et de mobilité des étudiants ou des chercheurs, sont autant de valeurs que promeut l'Université de Liège et qui en fondent la spécificité.

Les relations avec les entreprises sont également mises en avant. L'Université, dans ce cas, s'affiche en tant que partenaire potentiel des entreprises avec qui elle entend collaborer19. Il s'agira alors, pour les universités, d'associer [...] les entreprises innovantes, de se doter d'une mission en charge des

18. Dans le corpus, ces termes ont, pour la plupart d'entre eux, une fréquence supérieure à 20. Les formes graphiques (en lexicométrie, chaîne de caractères séparée par deux délimiteurs) ayant une fréquence supérieure à 20 correspondent à $3 \%$ du lexique, soit 106 formes (mots vides compris) sur 3392.

19. Pour une discussion sur l'importance du partenariat dans la redéfinition des universités, voir Moriau (2001). 
relations globales avec les entreprises, de consacrer la moitié des budgets de recherche [...] à des projets de collaboration avec les entreprises, leur permettant d'innover, de se développer et d'accroître leur compétitivité, d'associer des services scientifiques universitaires à un ensemble d'entreprises et d'acteurs économiques autour d'une thématique déterminée, etc. En outre, l'Université peut se concevoir elle-même sur le mode entrepreneurial :

(9) Université de Rennes 1 : la volonté de progresser, d'innover et d'entreprendre.

Dans d'autres cas, on met en avant la création d'entreprises spin-off, conséquence de la valorisation des activités de recherche, voire d'enseignement. L'université de Liège indique par exemple qu'elle est

(10) [...] encore pionnière dans la mise en place d'une politique cohérente et d'outils professionnels de valorisation de la recherche dont les résultats les plus visibles sont la création d'entreprises spin-off - plus de 80 sociétés créées à ce jour! - et un important portefeuille de technologies licenciables.

Les différents partenaires évoqués à travers le corpus reflètent également les parties prenantes (voir ci-dessous) qui sont désormais valorisées par les institutions: on retrouvera entre autres les collectivités territoriales / la ville / l'agglomération de communes / le conseil régional, les laboratoires, les pays développés ${ }^{20}$, les acteurs du monde économique / les entreprises, les structures partenaires, etc. L'université de Strasbourg souligne par exemple que venir étudier sur son campus, c'est opter «pour une plus forte ouverture sur la cité, le monde industriel et socio-économique».

Enfin, le terme vie est également prégnant dans les hypertextes. On retrouve notamment les syntagmes formation tout au long de la vie, vie communautaire / universitaire / sur le campus / étudiante, vie de la société (à laquelle participe l'Université), vie professionnelle et vie internationale (de l'institution). Le potentiel argumentatif actualisé par vie est ici intéressant : une fois sa vie étudiante terminée, la prochaine étape pour l'étudiant consiste à transitervers sa vie professionnelle, avant qu'il ne se forme tout au long de la vie. L'Université suit donc l'étudiant où qu'il soit (en plus d'une formation intra muros s'ajoute une formation à distance) et propose de l'aider à s'orienter et s'insérer dans la vie professionnelle à n'importe quel moment de son existence. Ce faisant, l'université de Strasbourg, toujours, "a pour objectif de favoriser la mobilité des étudiants et des enseignants et de faciliter la formation tout au long de la vie [et] entend être une université fortement participative à la vie de la société et non refermée sur elle-même» (nous soulignons).

Ces quelques exemples témoignent, à notre avis, de la correspondance

20. L'énoncé en question, d'où est tiré ce syntagme, parle au contraire de coopération avec les "pays émergents» et non plus de partenariat. 
interdiscursive entre, d'une part, les énoncés de promotion institutionnelle et, d'autre part, les propositions disséminées précisément dans les textes européens.

\section{Destinataires ciblés}

Ces différents textes s'accordent à dire que l'Université contemporaine doit davantage s'ouvrir à l'extérieur. Ici, la notion de réseau, en vogue depuis l'avènement d'Internet ${ }^{21}$, prend toute son importance : à l'instar des entreprises, l'Université, ancrée dans un espace d'échanges "glocalisé »22, se doit d'intégrer différents réseaux qui mettent en relation toute une série de partenaires, souvent qualifiés de parties prenantes (ou stakeholders) ${ }^{23}$, tant institutionnels qu'individuels. Le développement et le maintien de ces alliances réticulaires sont valorisés par les administrateurs universitaires, ceux-ci estimant qu'elles débouchent sur des projets de travaux pertinents et s'inscrivent dans les missions de leur université, notamment celles relatives aux services à la communauté.

Affichant leur volonté de briser la tour d'ivoire qui les symbolisait jusqu'ici, les universités sondées, de cette manière, mettent en scène leur ouverture et, ce faisant, rendent les murs de l'Université davantage perméables au monde extérieur. Cette importance accordée aux différentes parties prenantes transparaît dans les pages d'accueil, à travers les segments hypertextuels qui citent explicitement les destinataires ciblés par les communicants. Avec Barats (2010), nous considérons que «la présence ou l'absence de [tels ou tels destinataires] témoignent de choix stratégiques en termes de communication ». Globalement, nous avons pu repérer, dans le corpus, au moins douze types de destinataires, tant internes qu'externes à l'institution, directement invoqués à l'aide de dénominations parfois variées et qui témoignent d'une relative hétérogénéité : les étudiants (nationaux et internationaux), les adultes en reprise d'étude, les anciens étudiants (alumni), les entreprises, les chercheurs (internationaux), les enseignants, les chercheurs d'emploi, les journalistes/médias, le personnel, les visiteurs, les bailleurs de fonds potentiels et enfin, plus rarement, les citoyens. À nouveau, l'ensemble de ces destinataires sont ceux qui

21. Le «réseau » est ainsi devenu un modèle d'organisation type pour les entreprises. Pour Boltanski et Chiapello (1999, p. 156), «la récupération du terme de réseau s’est opérée [...] par la recherche, dans les sciences sociales, de concepts pour identifier des structures faiblement, voire pas du tout, hiérarchiques, souples et non limitées par des frontières tracées a priori. [...] Dans un monde réticulaire, [la vie sociale] est faite dorénavant d'une multiplication de rencontres et de connexions temporaires, mais réactivables, à des groupes divers, opérées à des distances sociales, professionnelles, géographiques, culturelles éventuellement très grandes ».

22. Ce mot-valise, en vogue, réunit les termes globalisé et localisé.

23. Le terme, en anglais, est souvent repris tel quel dans les textes en français. Rappelons qu'il vient du discours économique et a été utilisé pour distinguer ceux qui sont concernés par la vie de l'entreprise (employés, clients, institutions publiques, etc.) sans en être actionnaires (shareholders). 
sont favorisés dans les textes européens et qui correspondent aux nouveaux stakeholders auxquels doivent désormais s'adresser les universités.

Toutefois, cette ouverture est souvent imprécise et semble répondre à des exigences décidées de l'extérieur plutôt qu'être décidée démocratiquement de l'intérieur. Ainsi, certaines occurrences n'ont pas de déterminant pour compléter l'adjectif ouvert : on ignore ainsi, dans ce cas, à qui ou à quoi s'ouvre l'Université. On constate également la présence de références à des entités génériques imprécises, comme le montrent ces quelques exemples relevés à partir des occurrences de la forme ouvert* : 'Université est ouverte à la cité, à d'autres partenaires, au monde économique, auxattentes du monde professionnel, aul sur le monde, sur les réalités politiques, sociales, technologiques et industrielles, vers la ville et le monde socio-économique, sur l'Europe, etc. Ainsi, seule la catégorie «étudiants » comporte une différenciation réellement poussée (élèves du secondaire, futurs bacheliers/licenciés, anciens étudiants, etc.), qui dénote une volonté de l'énonciateur de cibler, de façon précise, chaque public invoqué. À l'inverse, la catégorie «entreprises » n'est pas différenciée : elle renvoie donc à un (des ?) destinataire(s) explicitement invoqué(s), mais dont l'identité reste a priori floue et indéterminée.

\section{Discussion}

À travers ces trois exemples de régularités, nous avons désiré montrer que la valorisation discursive des universités sondées dépend notamment de l'interdiscours européen sur l'enseignement supérieur. À côté des régularités d'ordre générique, nous avons en effet détecté des échos interdiscursifs récurrents mobilisant des termes, formules et points de vue défendus dans les différents textes officiels européens, notamment les communications de la Commission dont les propositions rythment les politiques nationales en matière d'enseignement et de recherche en Europe.

Cette dépendance discursive envers l'interdiscours européen nous paraît coextensive de la dépendance effective des universités envers le marché commun de l'enseignement et de la recherche européen émergent : en ce sens, harmonisation de l'enseignement supérieur 24 et harmonisation du discours promotionnel semblent aller de pair. Ainsi, les énoncés promotionnels se distinguent

24. Si les systèmes d'enseignement supérieur français, belge, luxembourgeois et suisse divergent en de nombreux points, ils ont tous la particularité d'être contraints de s'adapter au processus de Bologne et, pour les trois premiers, de s'intégrer à la stratégie de Lisbonne. En outre, pour le cas de la France, il se peut que les différences historiques entre les grandes écoles et les universités (ces dernières considérées comme étant moins prestigieuses que les premières) se retrouvent être minimisées en surface, à l'avantage des secondes, par ce même recours aux éléments de langage européens dans leur discours institutionnel. Des recherches ultérieures pourraient corroborer cette hypothèse. 
des énoncés publicitaires qui, dans un univers entrepreneurial hautement concurrentiel, se doivent d'être les plus novateurs et originaux possible : quand on pourrait s'attendre à ce que ces énoncés exacerbent les spécificités propres aux institutions qu'ils sont censés singulariser, l'analyse révèle au contraire une standardisation du discours, tant au niveau du dire qu'au niveau du dit. Nous suggérons que cette standardisation, en actualisant des «éléments de langage » identiques et en valorisant des atouts (réels ou, le plus souvent, escomptés) 25 similaires à chaque institution (internationalisation de l'enseignement et de la recherche, professionnalisation des chercheurs, adéquation entre compétences et marché du travail, évaluations des activités universitaires, etc.), témoigne d'une surdétermination du discours par le contexte européen englobant. Si des différences nettes, quant au rôle et prestige des établissements, subsistent bel et bien entre les universités belges, luxembourgeoises, françaises et suisses (ne serait-ce que par leur histoire respective), nous insistons sur le fait que le discours de promotion étudié, à travers des mises en scène similaires, d'établissement en établissement, tend manifestement à lisser ces différences ${ }^{26}$.

Plus précisément, dans le cas qui nous concerne, nous suggérons que le matériel discursif des hypertextes étudiés se trouve être travaillé par l’idéologie néolibérale. Si le symptôme principal est le vocabulaire managérial qui s’y trouve disséminé, il est également révélateur qu'un tel discours promotionnel, dont certaines parties, comme nous l'avons vu, se révèlent être exogènes, ne laisse pas transparaître une Université indépendante. La notion d' "autonomie contrôlée» (Dardot, Laval, 2010, p. 309) rend compte de ce constat. Contrairement au programme libéral classique, dont l'objectif vise un désengagement de l'État 27 en matière socio-économique, la pensée néolibérale lui reconnaît un interventionnisme radical : en tant qu'acteur économique à part entière, son rôle revient notamment à créer les conditions favorables d'une libéralisation de secteurs d'activités pourtant étrangers à la logique de marché, tel que l'était l'enseignement supérieur. Le rôle de la Commission européenne est ici central : celle-ci délivre aux États membres les impulsions normatives qu'il s'agira d'opérationnaliser dans les différents systèmes d'enseignement supérieur en Europe. Ainsi, des dispositifs de contrôle, centralisés par l'État, ont pour but de s'assurer que les universités, dans un environnement concurrentiel,

25. Des décalages apparaissent nécessairement entre le discours - ainsi que les représentations afférentes de l’Université qu'il construit - et les pratiques réellement adoptées au sein des établissements.

26. Dans le prolongement de cette recherche exploratoire, il s'agira toutefois de détecter, en discours, les différences plus subtiles qui subsistent au-delà des similitudes rapportées. D'emblée, on peut dire que les traits les plus spécifiques mis en avant concernent les valeurs et l'histoire propres à l'institution promue, ainsi que ses particularités disciplinaires et géographiques. Rappelons que les hypertextes étudiés se limitent à ceux visant à présenter et valoriser l'institution dans sa globalité (module de présentation institutionnelle).

27. Notons que les universités sondées, «publiques», sont toutes (historiquement) financées par le législateur. 
s'adaptent bel et bien aux nouvelles contraintes de l'économie de la connaissance et répondent aux nouvelles exigences d'excellence qu'elle suppose : instances d'évaluation des chercheurs, agences d'évaluation de la qualité de l'enseignement, instances nationales ou régionales pour l'octroi de financements, listes de revues qualifiantes pour la reconnaissance de la production scientifique, listes de catégories professionnelles normées en vue de «l'identification des chercheurs et enseignants-chercheurs "produisant en recherche et valorisation" " (AERES) 28 , etc. La perte d'autonomie se repère également au niveau des relations qu'entretiennent les universités avec les entreprises : si, autrefois, l'industrie bénéficiait des retombées scientifiques a posteriori, elle tend désormais à dicter a priori les orientations que doivent prendre les recherches, ce qui conduit à un estompage de l'autonomie de la recherche scientifique : "Les processus de construction des identités, de contrôle de qualité de la production, d'attribution des ressources, de choix des objets de recherche sont soumis à des influences extérieures» (Moriau, 2001).

Ce nouveau paradigme redéfinit donc le rôle des universités mais surdétermine également la façon dont celles-ci, in fine, se présentent (en ligne) face aux différents destinataires ciblés. Ceci n'est pas sans conséquence pour les acteurs universitaires concernés : par exemple, l'existence même d'un discours promotionnel alimente et exacerbe, en retour, la perception d'une mise en concurrence entre institutions.

Pour conclure, le site web universitaire, produit du management académique, constitue peut-être la marque la plus explicite de la mutation de l'enseignement supérieur en Europe : il s'agit d'un outil de communication plus ou moins standardisé, réceptacle d'un discours promotionnel harmonisé mobilisant des éléments d'un interdiscours exogène, pour, in fine, devenir une plateforme ouverte à la comparaison d'institutions devenues concurrentes sur les plans régional, national mais également international. À travers ces hypertextes, et plus particulièrement les modules de promotion institutionnelle, c'est donc toute une vision de l'enseignement supérieur (francophone) qui se trouve être matérialisée, et la sémantique du mot université qui se trouve être ainsi collectivement révisée.

\section{Références}

BARATS Christine, 2009, "Textes numériques ou sur le numérique. Rhétorique de l'internationalisation et "attractivité" des universités parisiennes», J.-M. Defays, A. Englebert éd., Acteurs et contextes des discours universitaires, vol.II, Paris, L'Harmattan, p. 209-225.

28. Agence d'évaluation de la recherche et de l'enseignement supérieur (France). 
- 2010, «Rhétorique de l'accueil académique. Pour une lecture comparée des sites universitaires de l'Ile-de-France », Actes du XVIIe Congrès de la Sociétéfrançaise des sciences de l'information et de la communication, Dijon, p.391-399.

Boltanski Luc, Chiapello Ève, 1999, Le nouvel esprit du capitalisme, Paris, Gallimard.

BRUno Isabelle, 2008, À vos marques, prêts... cherchez! La stratégie européenne de Lisbonne, vers un marché de la recherche, Bellecombe-en-Bauges, Croquant.

BRuno Isabelle, CLÉment Pierre, LAVAl Christian, 2010, La grande mutation. Néolibéralisme et éducation en Europe, Paris, Syllepse.

Commission européenne, 2003, Le rôle des universités dans l'Europe de la Connaissance, Bruxelles, Commission européenne.

Cussó Roser, 2008, "Quand la Commission européenne promeut la société de la connaissance », Mots. Les langages du politique, no 88, p. 39-52.

DARDot Pierre, LAVAL Christian, 2010, La nouvelle raison du monde. Essai sur la société néolibérale, Paris, La Découverte.

Defays Jean-Marc, 2009, "Défense et illustration de l'analyse des discours universitaires », J.-M. Defays, A. Englebert éd., Principes et typologie des discours universitaires, vol. I, Paris, L'Harmattan, p. 9-22.

KRIEg-PlAnque Alice, 2009, La notion de "formule» en analyse du discours. Cadre théorique et méthodologique, Besançon, Presses universitaires de Franche-Comté.

Krieg-Planque Alice, Oger Claire, 2010, «Discours institutionnels. Perspectives pour les sciences de la communication», Mots. Les langages du politique, n ${ }^{\circ} 94$, p. 91-96.

LIBAERT Thierry, JohANn ES Karine, 2010, La communication corporate, Paris, Dunod. Maingueneau Dominique, 1984, Genèses du discours, Bruxelles, Mardaga.

Moriau Jacques, 2001, "L'industrialisation de la recherche», J. Allard, G. Haarscher, M. Puig de la Bellacasa éd., L'université en questions. Marché des savoirs, nouvelle agora, tour d'ivoire?, Bruxelles, Labor, p. 50-77.

ReAdIngs Bill, 1996, The University in Ruins, Cambridge, Harvard University Press. 